\title{
The Role of Abnormal Methylation of Wnt5a Gene Promoter Regions in Human Epithelial Ovarian Cancer: A Clinical and Experimental Study
}

\author{
Ping Jin $\left(\mathbb{D},{ }^{1}\right.$ Yi Song, ${ }^{2}$ and Guiyuan $\mathbf{Y u}^{1}$ \\ ${ }^{1}$ The Shenzhen Maternity \& Child Healthcare Hospital Affiliated to Southern Medical University, ShenZhen 518028, China \\ ${ }^{2}$ The First Clinical Medical College of Lanzhou University, Lanzhou, China
}

Correspondence should be addressed to Ping Jin; 13925206896@163.com

Received 28 October 2017; Revised 10 February 2018; Accepted 26 February 2018; Published 16 July 2018

Academic Editor: Consuelo Amantini

Copyright (๑) 2018 Ping Jin et al. This is an open access article distributed under the Creative Commons Attribution License, which permits unrestricted use, distribution, and reproduction in any medium, provided the original work is properly cited.

\begin{abstract}
Objective. In the current study, the role of abnormal methylation of Wnt5a gene promoter regions in human epithelial ovarian cancer was investigated. Methods. Wnt5a expressions were examined by immunohistochemistry in epithelial ovarian tissues (30 normal and 79 human EOC tissues). SKOV3 cells were treated with different concentrations of 5-Aza-CdR (0.5, 5, and $50 \mu \mathrm{mol} / \mathrm{L})$. The methylation status of the Wnt5a promoter was analyzed using a methylation-specific polymerase chain reaction (MSP), and the expression level of Wnt5a mRNA was detected using quantitative real-time polymerase chain reaction (qRT-PCR). Cell proliferation was measured by MTT assay, and apoptosis was analyzed using flow cytometry. Results. (1) Compared with normal tissues, Wnt5a expressions were reduced or lost in EOC $(P<0.05)$. Wnt5a expression had a close relationship with histological grade, FIGO stage, and lymph node metastasis $(P=0.005, P=0.022$, and $P=0.037$, resp.). (2) Wnt5a abnormal methylation status existed in ovarian cancer tissues and was higher than that of normal ovarian tissue $(P<0.01)$. (3) Before treatment with 5-Aza-CdR, the promoter of the Wnt5a gene was methylated in SKOV3 cells; accordingly, Wnt5a mRNA levels were low to absent in SKOV3 cells. (4) Following 5-Aza-CdR treatment, MSP analysis revealed complete demethylation of the Wnt5a promoter in the SKOV3 cell line, particularly at $5 \mu \mathrm{mol} / \mathrm{L} 5$-Aza-CdR. Wnt5a expression increased in SKOV3 cells following treatment with a demethylating agent $(P \leq 0.001)$. (5) The growth rate of the cells was inhibited in a dose-dependent manner by treatment with 5 -Aza-CdR. (6) The cell apoptosis rate increased gradually after treatment with $0.5,5$, and $50 \mu \mathrm{mol} / \mathrm{L} 5$-Aza-CdR. The apoptosis rate exists in a dose-dependent relationship with 5-Aza-CdR concentration $(F=779.73, P<0.01)$. Conclusions. Wnt5a gene region promoter aberrant methylation existed in epithelial ovarian cancer, and abnormal methylation of Wnt5a gene promoter regions may be a new target for the treatment of epithelial ovarian cancer.
\end{abstract}

\section{Introduction}

The characteristics of ovarian cancer include a difficult early diagnosis, rapid development, and high mortality. More than $70 \%$ of ovarian cancer patients are diagnosed at an advanced stage, and the five-year survival rate is only $20 \%$. As a result, ovarian cancer is the most lethal malignant tumor of all female reproductive system tumors; epithelial ovarian cancer (EOC) is the most common type and accounts for approximately $60 \%-90 \%$ of all ovarian cancers [1]. As a result of in-depth studies in recent years, abnormalities of epigenetic modifications have been found to be one of the important reasons for tumor formation, are involved in the occurrence and development process of tumors, and are closely related to some pathologic types and prognosis. Epigenetic modification includes DNA methylation, microRNA regulation abnormalities, and histone acetylation. Among the modifications, DNA methylation is one of the most important methods of epigenetic regulation, which can cause changes to chromatin structure, DNA conformation, DNA stability, DNA-protein interaction, and gene expression. Abnormal promoter methylation is the molecular basis of genomic 
instability, and abnormal gene expression of the methylation status of tumor-related genes is an early sensitive indicator of tumor development [2, 3].

Wnt signals are involved in adult cell proliferation, differentiation, and apoptosis. The signal transduction pathways include the classical Wnt pathway, nonclassical Wnt/JNK pathway, and $\mathrm{Wnt} / \mathrm{Ca}^{2+}$ pathway; abnormal signal transduction pathways lead to tumor formation [4]. The Wnt5a gene, an important member of the Wnt family, is located on chromosome 3p, 14.2 p21.1. Wnt5a was firstly discovered by Clark et al., a molecular biologist at the University of Thomas Jefferson. The Wnt5a gene is composed of 1172 adenines, 884 cytosines, 946 guanines, and 1172 thymines. The gene contains 5 exons, and its terminal exon encodes the large $3^{\prime}$ end of the untranslated region. The promoter region is located in a region that is rich in glycerol phosphate choline and comprises many cis-acting elements $[5,6]$. The 631 base pair Wnt5a gene initiation region contains strong promoter activity [7]. Wnt5a is increased, decreased, or deleted in different tumors and, as a result, plays different roles in tumors [8]. DNA methylation is a reversible epigenetic modification. The DNA methyltransferase inhibitor 5-aza- $2^{\prime}$-deoxycytidine (5-Aza-CdR) inhibits the methylase enzyme DNMT, reverses hypermethylation of the promoter region to enable reexpression of tumor-associated genes, and inhibits tumor cell growth. In conclusion, the use of demethylating agents for cancer has become prominent in recent years [9].

The objective of this study was to understand the relationship between Wnt5a promoter methylation and epithelial ovarian cancer, observe the methylation status of the promoter region of the Wnt5a gene, explore the change in transcriptional expression and cell biological characteristics when treating with the demethylating agent 5-Aza-CdR, and finally provide a mechanistic basis to support the use of demethylating agents in the treatment of epithelial ovarian cancer.

\section{Materials and Methods}

2.1. Patients and Tissue Samples. Ninety-nine patients in the First Affiliated Hospital of Lanzhou University, China, from January 2009 to November 2013 were recruited including 79 patients of EOC and 30 cases of normal ovarian tissues The median age was 48 years (range from 27 to 73 years old). The experimental group comprised 79 untreated patients at different clinical stages: stage I $(n=15)$, stage II $(n=21)$, stage III $(n=29)$, and stage IV $(n=14)$. All the EOC patients were confirmed by histopathology including 50 serous adenocarcinomas, 23 mucinous adenocarcinomas, 4 endometrioid carcinomas, 1 Brenner's disease, and 1 clearcell carcinoma. Their histological grades were G1 $(n=13)$, G2 $(n=29)$, and G3 $(n=37)$. Diagnosis was confirmed or excluded based on standard morphologic, cytochemical, and immunophenotypic criteria. Informed consent was obtained from all the participants.

2.2. Immunohistochemistry. Immunostaining was performed with paraffin-embedded sections that were cut at $4 \mu \mathrm{m}$ and mounted onto glass slides. Tissue sections were deparaffinized with xylene and rehydrated through graded alcohol. Then, the slides were incubated in $3 \% \mathrm{H}_{2} \mathrm{O}_{2}$ for $10 \mathrm{~min}$ at room temperature so as to blocking endogenous peroxidase activity. Thereafter, antigen retrieval was performed in the citric acid repair solution for 5 seconds, $1700^{\circ} \mathrm{C}$, then stewed 5 minutes, and finally rinsed with PBS. Finally, the sections were incubated with primary antibody to Wnt5a (1:500 dilutions, ab86720; Abcam, UK) overnight at $4^{\circ} \mathrm{C}$. The next day, slides were stained with $\mathrm{DAB}$ and then counterstained applying hematoxylin. Wnt5a staining was shown in both the cytoplasm and the cytomembrane. The evaluation criterion of Wnt5a expression consists of both the staining intensity and the percentage of positive tumor cells. The former was graded as 0 to 3 (0: negative, 1: weak, 2: medium, and 3: strong). The latter was scored based on the percentage of the positive staining area in the total tumor area as follows: $0(<1 \%), 1(\leq 25 \%), 2(26 \%-50 \%), 3(51 \%-75 \%)$, and 4 $(>75 \%)$. These two scores were summed up as final staining scores (0-7). The staining score of $0-2$ was defined as low expression, 3-4 was called moderate expression, and 5-7 represented high expression. Thyroid cancer tissue was assigned as a positive control. On the contrary, the negative control was incubated with phosphate-buffered saline (PBS) alone instead of primary antibody.

2.3. Cell Culture and Drug Treatment. The SKOV3 ovarian cancer cell line was obtained from the Basic Medical Centre of Lanzhou University. The cells were maintained in RPMI 1640 supplemented with $10 \%$ FBS and $50 \mu \mathrm{g} / \mathrm{mL}$ gentamicin in a humidified $5 \% \mathrm{CO}_{2}$ atmosphere at $37^{\circ} \mathrm{C}$. The SKOV3 tumor cells $\left(2 \times 10^{5}\right.$ cells $)$ were cultured for three days in the presence of various concentrations $(0.5 \mu \mathrm{mol} / \mathrm{L}, 5 \mu \mathrm{mol} /$ $\mathrm{L}$, and $50 \mu \mathrm{mol} / \mathrm{L}$ ) of 5 -aza- $2^{\prime}$-deoxycytidine (5-Aza-CdR; Sigma, St. Louis, MO, USA). The cells were then cultured for an additional seven days in fresh culture medium without drugs to eliminate the influence of drug cytotoxicity. The control group was cultured in the absence of any agent.

2.4. Bisulphite Modification and Methylation-Specific PCR (MSP). Bisulphite modification of genomic DNA was evaluated using the CpG genome DNA Methylation-Gold ${ }^{\mathrm{TM}}$ Kit (Zymo Research, Irvine, CA, USA). The quality and integrity of the DNA were determined by the A260/280 ratio. The primer sequences for MSP amplification were Wnt5a-MD (5'-GTATTTTTCGGAGAAAAAGTTATGC- ${ }^{\prime}$ ) and Wnt5aMR (5'-ACAACCGCGAATTAATATAAACG- $\left.3^{\prime}\right)$ for the methylated reaction and Wnt5a-UD ( $5^{\prime}$-GGTATTTTTTG GAGAAAAAGTTATGTG- $3^{\prime}$ ) and Wnt5a-UR ( $5^{\prime}$-CTAC AACCACAAATTAATATAAACATC- $3^{\prime}$ ) for the unmethylated reaction [10]. "Hot start" PCR was performed for 35 cycles, consisting of denaturation at $95^{\circ} \mathrm{C}$ for $1 \mathrm{~min}$, annealing at $60^{\circ} \mathrm{C}$ for $1 \mathrm{~min}$, and extension at $72^{\circ} \mathrm{C}$ for $1 \mathrm{~min}$, followed by a final $7 \mathrm{~min}$ extension for both primer sets. The reaction products were separated by electrophoresis on $2 \%$ agarose gels. The DNA from blood marrow mononuclear cells treated with SssI methyltransferase (New England Biolabs, Ipswich, MA, USA) was used as a positive control for methylation. The results were confirmed by repeating 
the MSP assays following an independently performed bisulphite treatment.

\subsection{Quantitative Real-Time Polymerase Chain Reaction} ( $q R T-P C R)$. Total RNA was isolated from the SKOV3 ovarian cell line using TRIzol (Shanghai Sangon Biological Engineering Technology \& Services Co., China). All RNA samples were treated with TURBO DNase enzyme (TURBO DNA free kit; Ambion Inc., Applied Biosystems) to remove any contaminating DNA. Quantitative RT-PCR was performed using Takara Real-Time PCR System (Tokyo, Japan). The first-strand cDNA sample was then amplified using previously published primer sets for Wnt5a ( $5^{\prime}$-GTGCAATGT CTTCCAAGTTCTTC-3' (top strand) and $5^{\prime}$-GGCACAG TTTCTTCTGTCCTTG-3' (bottom strand)) [10] and $\beta$ actin ( $5^{\prime}$-GCATGGGTCAGAAGGATTCTT-3' (top strand) and $5^{\prime}$-TCGTCCCAGTTGGTGACGAT-3' (bottom strand)).

2.6. MTT Assay. Cells from the 5-Aza-CdR-treated or control groups were seeded in a 96-well plate at a density of $3 \times 10^{3}$ cells/well and incubated for $7 \mathrm{~d}$. Wells containing culture medium but with no cells were used as blanks. Cells were incubated with $20 \mu \mathrm{L}$ of $0.5 \mathrm{mg} / \mathrm{mL}$ MTT (Sigma) for $4 \mathrm{~h}$ at $37^{\circ} \mathrm{C}$. After removing the medium, formazan crystals were resolubilized with $150 \mu \mathrm{L}$ of dimethyl sulfoxide (Sigma) for $10 \mathrm{~min}$ at $37^{\circ} \mathrm{C}$. Absorbance at $570 \mathrm{~nm}$ was measured with a microplate reader. All test conditions were evaluated in triplicate wells on the same plates, and each experiment was repeated three times.

2.7. Annexin V and PI Double Staining of SKOV3 Apoptosis. SKOV3 cells from the 5-Aza-CdR-treated or control groups were resuspended at $6 \times 10^{5}$ cells $/ \mathrm{mL}$, and $1 \mathrm{~mL}$ of this suspension was seeded into cell culture flasks. The culture medium was removed once cells adhered to the surface, and then 5 -Aza-CdR was added to a final concentration of $0.5,5$, or $50 \mu \mathrm{mol} / \mathrm{L}$ in $10 \mathrm{~mL}$ of medium. For the control group, an equivalent volume of culture medium without drugs was added. The cells were incubated for $48 \mathrm{~h}$, collected, and resuspended in $0.5 \mathrm{~mL}$ of PBS, and then annexin $\mathrm{V}$ and PI were added. Cells were incubated in the dark for $10 \mathrm{~min}$ at room temperature and analyzed by flow cytometry.

2.8. Statistical Analysis. Quantitative data are reported as the mean \pm standard deviation (SD). Pearson's $\chi^{2}$ test was used to analyze the relationships between Wnt5a immunohistochemical staining and clinicopathologic variables. Contingency table analysis and Pearson's $\chi^{2}$ test were applied to compare the Wnt5a methylation status among cases and between various clinicopathologic variables. The difference in Wnt5a mRNA expression before and after 5-Aza-CdR treatment was analyzed by a paired sample $t$-test. The percentage of apoptosis was evaluated by the LSD test. All statistical tests were two-sided and were performed at the 5\% level of significance using the SPSS19.0 software package; $P<0.05$ was considered significant.

\section{Results}

3.1. Wnt5a Expression by Immunohistochemistry in Normal Ovary Tissues and EOC. Staining of Wnt5a was presented mainly in the cytoplasm. As shown in Figure 1, Wnt5a expression was significantly higher in normal ovaries $(21 / 30)$ than in epithelial ovarian carcinomas $(35 / 79)\left(P=0.017 ; \chi^{2}=5.747\right)$.

3.2. Methylation of Wnt5a in Human Epithelial Ovarian Carcinoma Tissues. MSP was used for the analysis of the Wnt5a methylation status (Figure 2). 31 of $79(39.24 \%)$ methylation were tested in EOC, while only 3 of $30(10 \%)$ was found in normal ovarian tissues. The difference was statistically significant $(P<0.01)$.

3.3. The Relationship between Wnt5a Expression and Methylation with Clinicopathological Features of EOC. From Table 1, Wnt5a expression was related to the histological grade, FIGO stage, and lymph node metastasis $(P=0.005$; $P=0.006$; and $P=0.037$, resp.). However, no significant difference was observed in other factors such as age and histological type.

For the part of methylation, statistical analysis showed no significant correlation between promoter region hypermethylation and age, histological type, or WHO grades. On the contrary, we observed an obvious relation between promoter region hypermethylation with FIGO stages and lymphatic metastasis $(P=0.018$ and $P=0.024$, resp.). The methylation was discovered in 9 of 36 (25\%) FIGO stage I II and in 22 of 43 (51.16\%) FIGO stage III IV; Wnt5a was methylated in 21 of 41 (51.22\%) carcinomas with lymph node metastasis, while 10 of $28(26.32 \%)$ tumors without lymph node metastasis.

\subsection{Methylation of Wnt5a in the Human Epithelial Ovarian} Cancer Cell Line SKOV3. The methylation status of Wnt5a in the human epithelial ovarian cancer cell line SKOV3 was determined by MSP analysis (Figure 3). The band corresponding to the methylated $W n t 5 a$ gene is indicated (107bp). The primers recognizing the unmethylated gene were able to amplify a band of weaker intensity, suggesting that $W n t 5 a$ is partially methylated in this cell line.

3.5. 5-Aza-CdR Treatment Induces Demethylation of the Wnt5a Promoter and Increases mRNA Expression in the SKOV3 Cell Line. SKOV3 cells were treated with different concentrations of the demethylating agent $5-\mathrm{Aza}-\mathrm{CdR}$ to determine whether methylation of the $W n t 5 a$ promoter is the basis for the low level of expression. The MSP analysis revealed complete demethylation of the Wnt5a promoter in the SKOV3 cell line following 5-Aza-CdR treatment (Figure 4).

The results of mRNA expression after 5-Aza-CdR treatment are shown in Figure 5, which represented that $W n t 5 a$ expression was increased in SKOV3 cells following treatment with the demethylating agent $(P \leq 0.001)$. These data indicated that promoter methylation was closely correlated with low levels of Wnt5a expression in the SKOV3 cell line. 


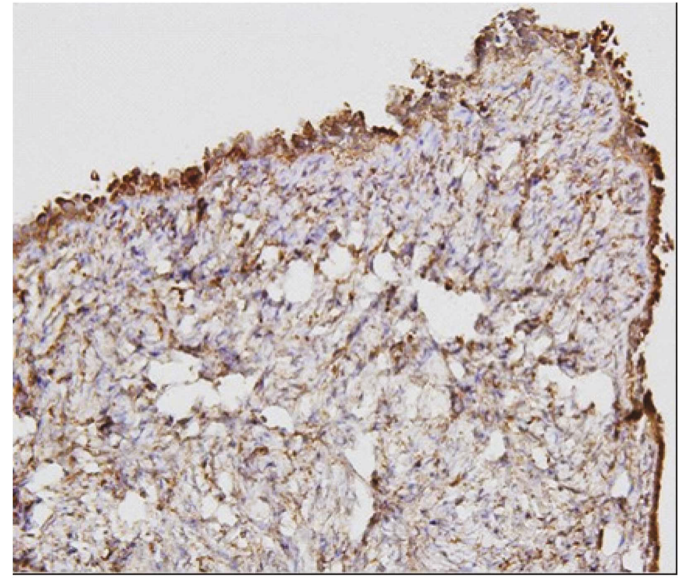

(a)

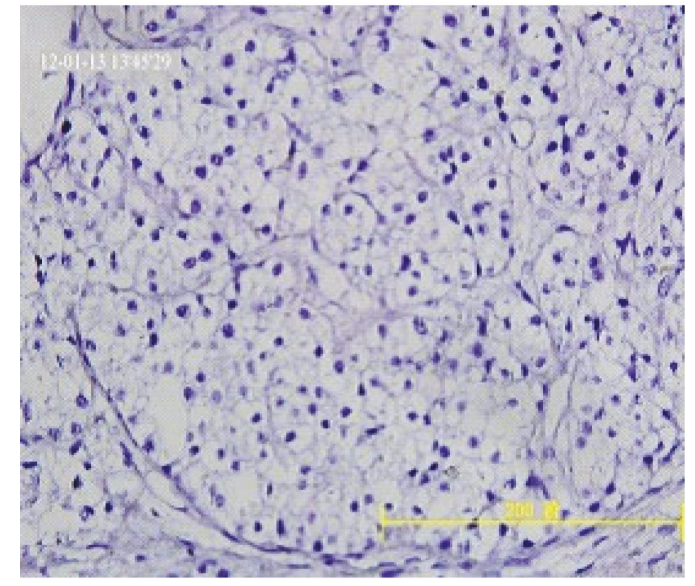

(b)

FIGURE 1: Expression of Wnt5a in normal ovarian tissue and ovarian cancer. Normal ovaries with immunostaining of Wnt5a in the cytoplasm of tumor cells. Staining index $(\mathrm{SI}=7)$ in epithelial ovarian tissues. Immunolabeling in the cytoplasm of fibroblasts on the (a) (40x). Ovarian cancer was clear-cell cancer, in the cytoplasm of tumor cells $(40 x$; SI $=0)$.

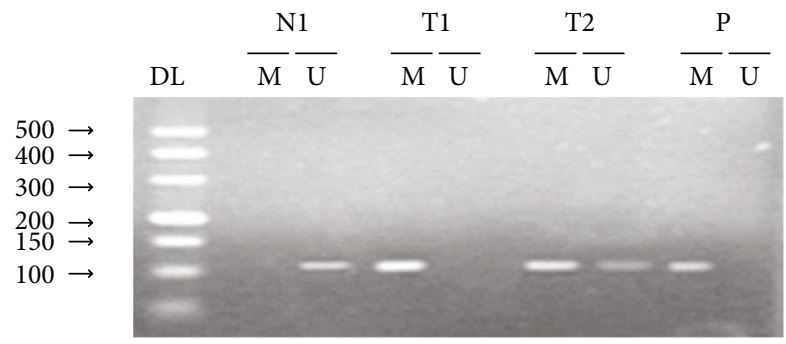

Figure 2: Methylation status of Wnt5a in normal ovarian tissues and epithelial ovarian carcinomas. N: normal ovarian tissues; T: epithelial ovarian carcinomas; P: positive control; DL500: DNA marker; M: methylation; U: unmethylated.

3.6. The Impact of 5-Aza-CdR Treatment on the Growth Rate of SKOV3 Cells. The growth rate of the cells was inhibited in a dose-dependent manner by treatment with 5-Aza-CdR. In the first $96 \mathrm{~h}$, we found that SKOV3 cell growth was inhibited in different concentrations of 5 -Aza-CdR. And the $50 \mu \mathrm{mol} / \mathrm{L}$ 5 -Aza-CdR has the higher effects than other groups. But after $96 \mathrm{~h}$, all drugs' group changed subtly, but it is strange that the inhibition of $50 \mu \mathrm{mol} / \mathrm{L} 5$-Aza-CdR was attenuated (Figure 6).

3.7. The Influence of 5-Aza-CdR on the SKOV3 Cell Apoptosis Rate. By flow cytometry analysis, the cell apoptosis rate increased gradually after treatment with $0.5,5$, and $50 \mu \mathrm{mol} /$ L 5-Aza-CdR; the apoptosis rates were $4.24 \% \pm 0.79 \%$, $9.20 \% \pm 0.26 \%$, and $20.48 \% \pm 1.89 \%$, respectively, compared with the control group $(1.89 \% \pm 0.48 \%)(P<0.01)$. The apoptosis rate and 5-Aza-CdR concentration show a dosedependent relationship $(F=779.73, P<0.01)$ (Figure 7$)$.

\section{Discussion}

DNA methylation refers to the catalytic action of DNA methyltransferase (DNMT1, DNMT3A, and DNMT3B), which can covalently combine the S-adenosylmethionine (SAM) to the $\mathrm{CpG}$ dinucleotide 5-carbon position of cytosine to result in the formation of 5-methylcytosine using sadenosylmethionine (SAM) as a methyl donor [11]. CpG dinucleotides are the major site of DNA methylation, which is nonrandomly distributed throughout the genome. Typically, DNA sequences with a $\mathrm{CpG}$ content that is more than $50 \%$ and length that is greater than $200 \mathrm{bp}$ are called $\mathrm{CpG}$ islands (CpG islands (CGIs)) [12]. In mammals, DNA methylation occurs mostly in the $\mathrm{CpG}$ island cytosine, playing an important role in the regulation of gene expression. A considerable part of methylation occurs outside the $\mathrm{CpG}$ island region (non-CpG site), but the role of this methylation is not clear $[13,14]$. CpG islands are mainly located in the promoter region of the gene, typically in a nonmethylated form, but when tumors occur, in particular, hypermethylation occurs in the CpG islands of tumor suppressor genes (TSG) $[15,16]$.

The Wnt5a gene is an important member of the Wnt family. Researchers have found that Wnt5a can be involved in the occurrence and development of tumors by various manners. Wnt5a can affect cell migration, invasion, and angiogenesis in tissues; however, it is also conducive to tissue repair and maintenance of the steady state of tissues [16]. Some studies demonstrated that Wnt5a functions through the Wnt/Ca ${ }^{2+}$ pathway in some tumors. Wnt5a can not only activate but also inhibit the canonical $\mathrm{Wnt} / \beta$-catenin pathway, depending on what type of receptor $W n t 5 a$ combines with. When $W n t 5 a$ binds to Ror2, the canonical Wnt/ $\beta$ catenin pathway is being inhibited by the $\mathrm{Wnt} / \mathrm{Ca}^{2+}$ pathway. However, when Wnt5a binds to Frizzled and LRP, the opposite effect occurs. Thus, whether Wnt5a suppresses or leads to cancer formation depends on the cell surface receptor it binds [17-19]. In addition, expression of the Wnt5a gene may be increased, decreased, or absent in tumors. Wnt5a methylation also differs among tumors, and the mechanism of Wnt5a methylation in tumor occurrence and development differs. As a result, Wnt5a plays dual roles. In some tumors, such as melanoma, gastric cancer, osteosarcoma, prostate 
TABLE 1: Relationship between Wnt5a immunohistochemistry and methylation with clinicopathologic features of ovarian carcinoma.

\begin{tabular}{|c|c|c|c|c|c|}
\hline Clinicopathologic parameters & $n$ & Protein expression & $P$ & Methylation positive & $P$ \\
\hline \multicolumn{6}{|l|}{ Age (years) } \\
\hline$\leq 48$ & 34 & $14(41.18)$ & \multirow{2}{*}{$>0.05$} & $13(38.24)$ & \\
\hline$>48$ & 45 & $21(46.47)$ & & $18(40)$ & $>0.05$ \\
\hline \multicolumn{6}{|l|}{ WHO grades } \\
\hline G1 & 13 & $10(76.92)$ & \multirow{3}{*}{$0.005^{*}$} & $5(38.46)$ & \\
\hline G2 & 29 & $13(44.83)$ & & $10(34.48)$ & $>0.05$ \\
\hline G3 & 37 & $12(32.43)$ & & $16(43.24)$ & \\
\hline \multicolumn{6}{|l|}{ FIGO stages } \\
\hline $\mathrm{I} \sim \mathrm{II}$ & 36 & $22(61.11)$ & \multirow{2}{*}{0.006} & $9(25)$ & \\
\hline III IV & 43 & $13(30.23)$ & & $22(51.16)$ & 0.018 \\
\hline \multicolumn{6}{|l|}{ Histological types } \\
\hline Serous adenocarcinoma & 50 & $22(44)$ & \multirow{3}{*}{$>0.05$} & $19(38)$ & \\
\hline Mucinous adenocarcinoma & 23 & $11(47.83)$ & & $9(39.13)$ & $>0.05$ \\
\hline Others & 6 & $3(50)$ & & $2(33.33)$ & \\
\hline \multicolumn{6}{|l|}{ Lymph node metastasis } \\
\hline No & 38 & $20(52.63)$ & \multirow{2}{*}{0.037} & $10(26.32)$ & \multirow[t]{2}{*}{0.024} \\
\hline Yes & 41 & $15(36.59)$ & & $21(51.22)$ & \\
\hline
\end{tabular}

*The $P$ value of G1 compared with that of G3.

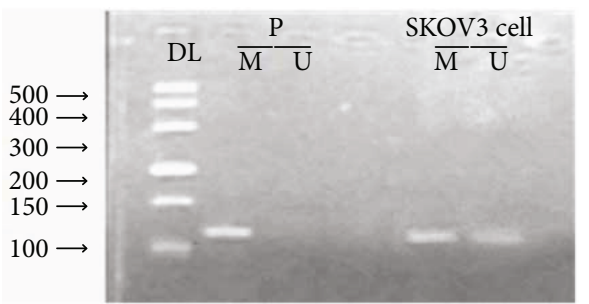

FIGURE 3: Methylation of Wnt5a in the human epithelial ovarian cancer cell line SKOV3. P: positive control; DL500: DNA marker; M: methylation; U: unmethylated.

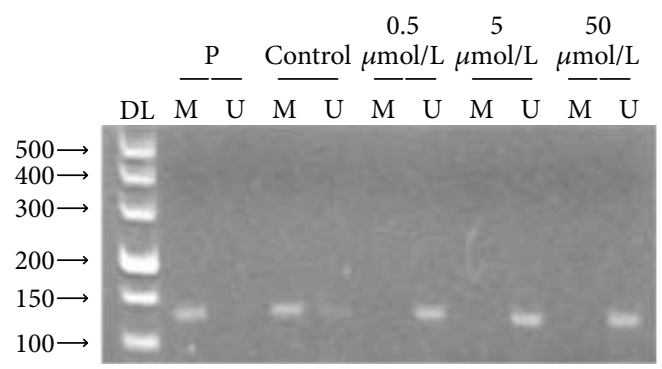

FIGURE 4: Demethylation of Wnt5a by different concentrations of 5-AzA-CdR in the SKOV3 cell line. DL500: DNA marker; $\mathrm{P}$ : positive control $\mathrm{M}$ : methylation; $\mathrm{U}$ : unmethylated.

cancer, nonsmall cell lung cancer, nasopharyngeal carcinoma, and pancreatic cancer, Wnt5a is overexpressed and plays the role of an oncogene [20-24]. In other tumors, such as colorectal cancer, esophageal squamous cell carcinoma, thyroid carcinoma, breast cancer, and white blood cell disease, $W n t 5 a$ shows low expression or loss of expression and plays the role of a tumor suppressor gene [25-28].
In our study, we described that Wnt5a expression was significantly higher in normal ovaries than in ovarian carcinomas with a proportion of $70 \%$ to $44.3 \%$. In addition, Wnt5a expression was related to the histological grade, FIGO stage, and lymph node metastasis but was not associated with age and histological type (Figure 1, Table 1). Bitler et al. [29] also found that $W n t 5 a$ gene expression levels were significantly lower in EOC patients, and low levels of Wnt5a expression were significantly related to tumor staging and predicted shorter overall survival in patients when compared with normal ovarian surface epithelial cells or fallopian tube epithelial cells. Notably, increased Wnt5a expression significantly inhibited the proliferation of human EOC cells and ultimately played a role in promoting cell senescence in EOC. This is consistent with our experimental results, supporting the possible tumor suppressor gene characteristics of Wnt5a in ovarian cancer. However, some scholars have found that when compared to a benign epithelial neoplasia group and normal ovary group, the proportion of Wnt5apositive women was significantly higher for the epithelial ovarian cancer group [30]. This result may not be consistent with the results of our study, but the results of our experiments have been demonstrated by both in vivo and in vitro experiments, and related research also found similar results. In addition, Bitler et al. [29] found that increased Wnt5a expression significantly inhibited the proliferation of human EOC cells. As a result, the differences in the conclusions of this study should be further studied and discussed to explain the reasons for these differences.

DNA methylation is widespread in epithelial ovarian tumors. Ho et al. [31] found that the methylation profiles of tumor suppressor genes (TSGs) were significantly higher in the ovarian cancer stromal progenitor cells (OCSPCs) than in ovarian cancer cells. OCSPCs and decreased TSG 


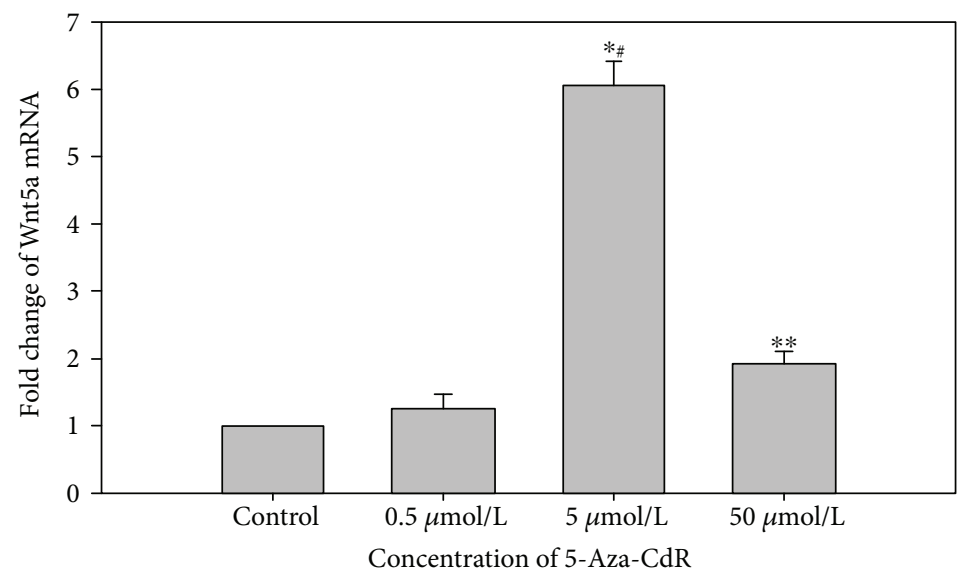

Figure 5: Expression of Wnt5a gene mRNA with different concentrations of 5-Aza-CdR in the SKOV3 cell line. ${ }^{\#}$ Compared with the control group, $\left({ }^{*} P<0.01,{ }^{* *}\right.$ different concentrations of 5 -Aza-CdR).

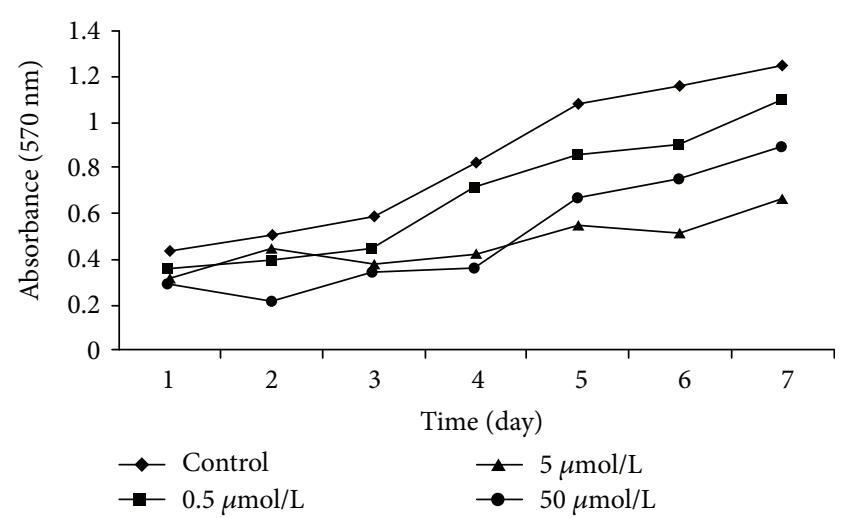

FIGURE 6: SKOV3 cell proliferation in a time- and dose-dependent manner.

expression in the ovarian tumor microenvironment were able to promote tumorigenesis, which could be reversed by DNA demethylation. In the current study, the methylation status of Wnt5a in the human epithelial ovarian cancer was determined by MSP analysis (Figures 2 and 3). Our data show that Wnt5a promoter region abnormal methylation did exist in EOC and was closely associated with clinical progression of EOC (Table 1). In addition, a study found that $\mathrm{CpG}$ island methylation in ovarian cancer is related to the silencing of many genes, including BRCA1, RASSF1A, LOT1 and Hmlh1; the silencing of these genes may be involved in the development of ovarian cancer [16]. The inactivation of tumor suppressor genes is related to hypermethylation, whereas genome-wide hypomethylation and local (CpG island) hypermethylation in tumors may be caused by disruptions of DNMT activity [32].

Our study further revealed complete demethylation of the Wnt5a promoter in the SKOV3 cell line following 5Aza-CdR treatment $(0.5,5$, and $50 \mu \mathrm{mol} / \mathrm{L})$ (Figure 4$)$. The expression of Wnt5a mRNA was increased (Figure 5), particularly at $5 \mu \mathrm{mol} / \mathrm{L} 5$-Aza-CdR. These data indicated that the hypermethylation status of the promoter region of the Wnt5a gene in SKOV3 cells was reversed and that the Wnt5a mRNA was reexpressed. DNA methylation is a reversible epigenetic modification process that can be reversed by demethylation. 5-Aza-2-deoxycytidine (5-Aza-CdR) is now widely recognized as a demethylation agent. 5-Aza-CdR inhibits the methylase enzyme DNMT and stops cell DNA replication. Then, the methyl can not be transferred to the cytosine, thereby reducing the DNA methyl-accepting ability to reduce the degree of methylation in the progeny of the cells; thus, the tumor suppressor gene, whose promoter region is hypermethylated, finally reverses the biological activity of tumor cells [10]. 5-Aza-2' -deoxycytidine treatment not only reactivates genes but also decreases the overexpression of genes [33]. The difference in the two concentrations of 5-Aza-2deoxycytidine (5-Aza-CdR) influences genome-wide methylation and induces changes in different sugar chains [34]. 5-Aza-CdR methylation inhibitors have been widely used in experimental studies of different tumors, such as colorectal cancer, breast cancer, thyroid cancer, and leukemia $[35,36]$.

In the current study, the growth rate of the tumor cells was inhibited by treatment with 5-Aza-CdR (Figure 6). A strange phenomenon in this result was that the inhibition of $50 \mu \mathrm{mol} / \mathrm{L} 5$-Aza-CdR was attenuated. Two reasons may explain this strange phenomenon; one is the widespread hypomethylation of the Wnt5a genome in all drugs' group. The other is that the drug of 5-Aza-CdR may have reached halflife and the effect starts to wane, which was supported by some researches [37]. The study suggested that 5-Aza-CdR can inhibit the proliferation of SKOV3 cells, and the inhibition was gradually increased with increasing drug concentration. This inhibition has been applied in many tumors. In breast cancer, DAC (5-aza-2'-deoxycytidine) treatment caused significant breast cancer stem cell (BCSC) differentiation. DAC reduced breast cancer cell survival and induced differentiation through reexpression of tumor suppressor genes [36].

In addition, our research showed that the cell apoptosis rate increased gradually after treatment with $0.5,5$, and $50 \mu \mathrm{mol} / \mathrm{L} 5$-Aza-CdR. The rate apoptosis and 5-Aza-CdR concentration share a dose-dependent relationship (Figure 7). 5-Aza-CdR efficiently induced cell cycle arrest at G0/G1 and apoptotic death in HXO-RB44 cells. An MSP analysis showed that unmethylated RASSF1A DNA increased and 


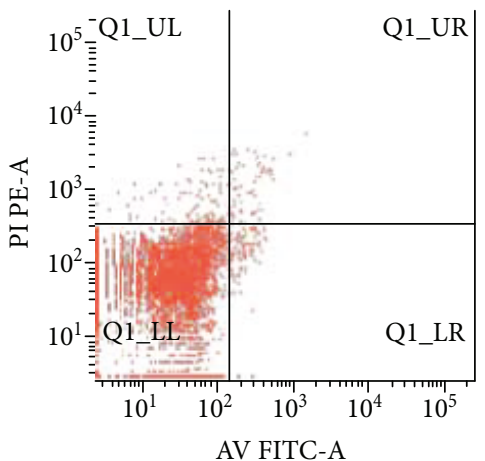

(a)

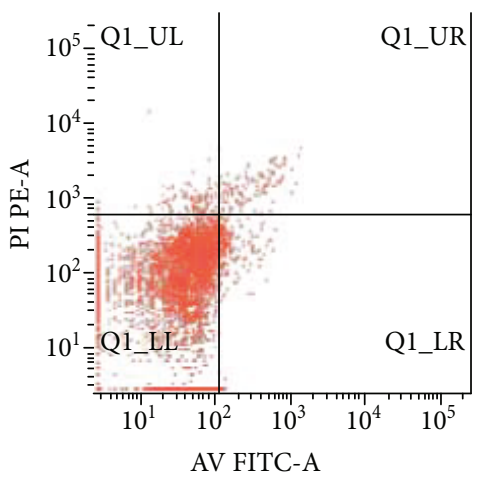

(c)

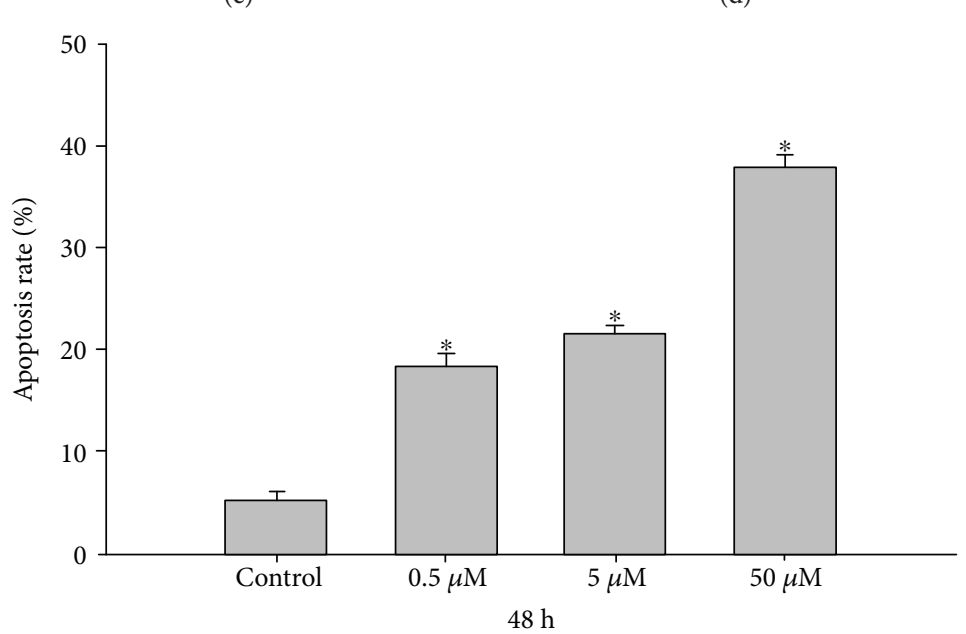

(e)

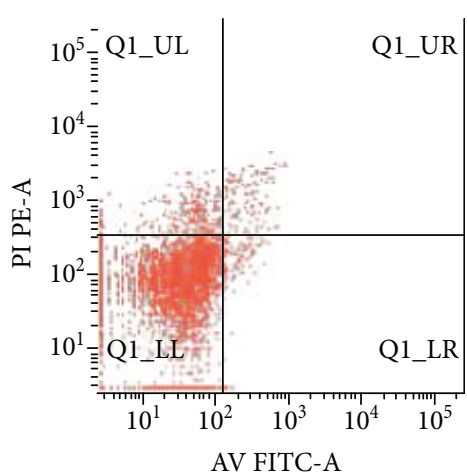

(b)

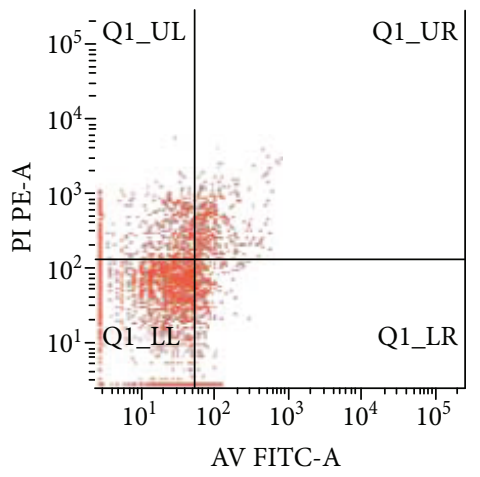

(d)

Figure 7: The rate of apoptosis and 5-Aza-CdR concentration show a dose-dependent relationship ( ${ }^{*} P<0.01$ ). (a) Control. (b) $0.5 \mu \mathrm{mol} / \mathrm{L}$ dose group. (c) $5 \mu \mathrm{mol} / \mathrm{L}$ dose group. (d) $50 \mu \mathrm{mol} / \mathrm{L}$ dose group. (e) The statistics of apoptosis rate in each group.

methylated RASSF1A decreased in a dose-dependent manner in a range of $0.5-5.0 \mu \mathrm{mol} / \mathrm{L} 5-\mathrm{Aza}-\mathrm{CdR}$. 5-Aza-CdR inhibits the growth of the HXO-RB44 RB cell line and induces apoptosis by demethylating the RASSF1A gene [38]. Several previous studies reported that 5-Aza-CdR exhibited cytotoxicity but the cells demonstrated cytotoxicity and that the effect was not hereditary [39]. In this experiment, we replaced 5-Aza-CdR with fresh medium for 7 days to avoid the toxic effects of 5-Aza-CdR. Therefore, the growth inhibition and apoptosis of cells in this experiment were a true reflection of 5-Aza-CdR demethylation.
In summary, Wnt5a gene region promoter aberrant methylation existed in epithelial ovarian cancer tissue, which is one of the important mechanisms of Wnt5a gene inactivation. 5-Aza-CdR treatment reversed the methylation status of the promoter and restored $W n t 5 a$ gene expression, which behaved as a tumor suppressor. Significantly, 5-Aza-CdR inhibited proliferation and induced apoptosis of SKOV3 cells due to the reversal of promoter hypermethylation of genes, including Wnt5a. Based on our results, Wnt5a demethylation may be a new target for the treatment of epithelial ovarian cancer. 


\section{Conflicts of Interest}

The authors confirm that this article has no financial or commercial conflicts of interest.

\section{Authors' Contributions}

Ping Jin and Yi Song contributed equally to this work.

\section{Acknowledgments}

This article was supported by the Science and Technology Innovation Committee Basic Research (subject layout) Fund of Shenzhen (Grant no. JCYJ20170413165233512) to Ping Jin.

\section{References}

[1] E. Lengyel, "Ovarian cancer development and metastasis," The American Journal of Pathology, vol. 177, no. 3, pp. 1053-1064, 2010.

[2] K. D. Robertson, "DNA methylation and human disease," Nature Reviews Genetics, vol. 6, no. 8, pp. 597-610, 2005.

[3] F. F. Costa, "Epigenomics in cancer management," Cancer Management and Research, vol. 2, pp. 255-265, 2010.

[4] J. Yu and D. M. Virshup, "Updating the Wnt pathways," Bioscience Reports, vol. 34, no. 5, article e00142, 2014.

[5] M. Kühl, "The WNT/calcium pathway: biochemical mediators, tools and future requirements," Frontiers in Bioscience, vol. 9, pp. 967-974, 2004.

[6] C. C. Clark, I. Cohen, I. Eichstetter et al., "Molecular cloning of the human proto-oncogene Wnt-5A and mapping of the gene (WNT5A) to chromosome 3p14-p21," Genomics, vol. 18, no. 2, pp. 249-260, 1993.

[7] M. G. Prieve and R. T. Moon, "Stromelysin-1 and mesothelin are differentially regulated by Wnt-5a and Wnt-1 in C57mg mouse mammary epithelial cells," BMC Developmental Biology, vol. 3, no. 1, p. 2, 2003.

[8] N. Chiba, K.-I. Furukawa, S. Takayama et al., "Decreased DNA methylation in the promoter region of the WNT5A and GDNF genes may promote the osteogenicity of mesenchymal stem cells from patients with ossified spinal ligaments," Journal of Pharmacological Sciences, vol. 127, no. 4, pp. 467-473, 2015.

[9] M. Daskalakis, T. T. Nguyen, C. Nguyen et al., "Demethylation of a hypermethylated P15/INK4B gene in patients with myelodysplastic syndrome by 5 -Aza- $2^{\prime}$-deoxycytidine (decitabine) treatment," Blood, vol. 100, no. 8, pp. 2957-2964, 2002.

[10] J. Roman-Gomez, A. Jimenez-Velasco, L. Cordeu et al., "WNT5A, a putative tumour suppressor of lymphoid malignancies, is inactivated by aberrant methylation in acute lymphoblastic leukaemia," European Journal of Cancer, vol. 43, no. 18, pp. 2736-2746, 2007.

[11] A. Jeltsch, "Beyond Watson and Crick:DNA methylation and molecular enzymology of DNA methyltransferases," ChemBioChem, vol. 3, no. 4, pp. 274-293, 2002.

[12] Z. D. Smith and A. Meissner, "DNA methylation: roles in mammalian development," Nature Reviews Genetics, vol. 14, no. 3, pp. 204-220, 2013.

[13] J. A. Law and S. E. Jacobsen, "Establishing, maintaining and modifying DNA methylation patterns in plants and animals," Nature Reviews Genetics, vol. 11, no. 3, pp. 204-220, 2010.
[14] W. Xie, C. L. Barr, A. Kim et al., "Base-resolution analyses of sequence and parent-of-origin dependent DNA methylation in the mouse genome," Cell, vol. 148, no. 4, pp. 816-831, 2012.

[15] H. K. Long, H. W. King, R. K. Patient, D. T. Odom, and R. J. Klose, "Protection of CpG islands from DNA methylation is DNA-encoded and evolutionarily conserved," Nucleic Acids Research, vol. 44, no. 14, pp. 6693-6706, 2016.

[16] W. J. Poh, C. P. P. Wee, and Z. Gao, "DNA methyltransferase activity assays: advances and challenges," Theranostics, vol. 6, no. 3, pp. 369-391, 2016.

[17] T. N. H. Masckauchán, D. Agalliu, M. Vorontchikhina et al., "Wnt5a signaling induces proliferation and survival of endothelial cells in vitro and expression of MMP-1 and Tie-2," Molecular Biology of the Cell, vol. 17, no. 12, pp. 5163-5172, 2006.

[18] Y. Yuan, C. C. Niu, G. Deng et al., “The Wnt5a/Ror2 noncanonical signaling pathway inhibits canonical Wnt signaling in K562 cells," International Journal of Molecular Medicine, vol. 27, no. 1, pp. 63-69, 2011.

[19] A. J. Mikels and R. Nusse, "Purified Wnt5a protein activates or inhibits $\beta$-catenin-TCF signaling depending on receptor context," PLoS Biology, vol. 4, no. 4, article e115, 2006.

[20] J.-z. Li, Y. Zhang, B. Wen, M. Li, and Y.-j. Wang, "Ability of PITX2 methylation to predict survival in patients with prostate cancer," OncoTargets and Therapy, vol. 8, pp. 3507-3512, 2015.

[21] K. Hibi, M. Sakata, K. Yokomizi et al., "Methylation of the WNT5A gene is frequently detected in early gastric carcinoma," Hepato-Gastroenterology, vol. 59, no. 120, pp. 26612663, 2012.

[22] E. J. Ekström, V. Sherwood, and T. Andersson, "Methylation and loss of secreted frizzled-related protein 3 enhances melanoma cell migration and invasion," PLoS One, vol. 6, no. 4, article e18674, 2011.

[23] H. Vaidya, C. Rumph, and K. S. Katula, "Inactivation of the WNT5A alternative promoter B is associated with DNA methylation and histone modification in osteosarcoma cell lines U2OS and SaOS-2," PLoS One, vol. 11, no. 3, article e0151392, 2016.

[24] H. Bo, L. Gao, Y. Chen, J. Zhang, and M. Zhu, "Upregulation of the expression of Wnt5a promotes the proliferation of pancreatic cancer cells in vitro and in a nude mouse model," Molecular Medicine Reports, vol. 13, no. 2, pp. 1163-1171, 2016.

[25] J. Li, J. Ying, Y. Fan et al., "WNT5A antagonizes WNT/ $\beta$ catenin signaling and is frequently silenced by promoter CpG methylation in esophageal squamous cell carcinoma," Cancer Biology \& Therapy, vol. 10, no. 6, pp. 617-624, 2010.

[26] J. B. Rawson, M. Mrkonjic, D. Daftary et al., "Promoter methylation of $W n t 5 a$ is associated with microsatellite instability and BRAF V600E mutation in two large populations of colorectal cancer patients," British Journal of Cancer, vol. 104, no. 12, pp. 1906-1912, 2011.

[27] Ö. Hatırnaz Ng, S. Fırtına, İ. Can et al., "A possible role for WNT5A hypermethylation in pediatric acute lymphoblastic leukemia," Turkish Journal of Hematology, vol. 32, no. 2, pp. 127-135, 2015.

[28] F. Trifa, S. Karray-Chouayekh, E. Jmal et al., "Loss of WIF-1 and Wnt5a expression is related to aggressiveness of sporadic breast cancer in Tunisian patients," Tumour Biology, vol. 34, no. 3, pp. 1625-1633, 2013. 
[29] B. G. Bitler, J. P. Nicodemus, H. Li et al., "Wnt5a suppresses epithelial ovarian cancer by promoting cellular senescence," Cancer Research, vol. 71, no. 19, pp. 6184-6194, 2011.

[30] L. Badiglian Filho, C. T. Oshima, F. De Oliveira Lima et al., "Canonical and noncanonical Wnt pathway: a comparison among normal ovary, benign ovarian tumor and ovarian cancer," Oncology Reports, vol. 21, no. 2, pp. 313-320, 2009.

[31] C. M. Ho, D. T. Shih, C. C. Hsiao, S. H. Huang, S. F. Chang, and W. F. Cheng, "Gene methylation of human ovarian carcinoma stromal progenitor cells promotes tumorigenesis," Journal of Translational Medicine, vol. 13, no. 1, p. 367, 2015.

[32] F. Ozdemir, J. Altinisik, A. Karateke, H. Coksuer, and N. Buyru, "Methylation of tumor suppressor genes in ovarian cancer," Experimental and Therapeutic Medicine, vol. 4, no. 6, pp. 1092-1096, 2012.

[33] X. Yang, H. Han, D. D. De Carvalho, F. D. Lay, P. A. Jones, and G. Liang, "Gene body methylation can alter gene expression and is a therapeutic target in cancer," Cancer Cell, vol. 26, no. 4, pp. 577-590, 2014.

[34] M. Klasić, J. Krištić, P. Korać et al., "DNA hypomethylation upregulates expression of the MGAT3 gene in HepG2 cells and leads to changes in $\mathrm{N}$-glycosylation of secreted glycoproteins," Scientific Reports, vol. 6, no. 1, article 24363, 2016.

[35] S. Yang, B. Wu, H. Sun et al., "Interrupted E2F1-miR-34c-SCF negative feedback loop by hyper-methylation promotes colorectal cancer cell proliferation," Bioscience Reports, vol. 36, no. 1, article e00293, 2016.

[36] N. L.-C. Phan, N. Van Trinh, and P. Van Pham, "Low concentrations of 5-aza-2' -deoxycytidine induce breast cancer stem cell differentiation by triggering tumor suppressor gene expression," OncoTargets and Therapy, vol. 9, pp. 49-59, 2016.

[37] Z. D. Zhivkova, T. Mandova, and I. Doytchinova, "Quantitative structure - pharmacokinetics relationships analysis of basic drugs: volume of distribution," Journal of Pharmacy \& Pharmaceutical Sciences, vol. 18, no. 3, pp. 515-527, 2015.

[38] R. Liu, X. H. Zhang, K. Zhang et al., "5-Aza-2'-deoxycytidine inhibits retinoblastoma cell by reactivating epigenetically silenced RASSF1A gene," International Journal of Ophthalmology, vol. 7, no. 1, pp. 51-56, 2014.

[39] J. M. D. Wheeler, N. E. Beck, H. C. Kim, I. P. M. Tomlinson, N. J. McC. Mortensen, and W. F. Bodmer, "Mechanisms of inactivation of mismatch repair genes in human colorectal cancer cell lines: the predominant role of hMLH1," Proceedings of the National Academy of Sciences of the United States of America, vol. 96, no. 18, pp. 10296-10301, 1999. 


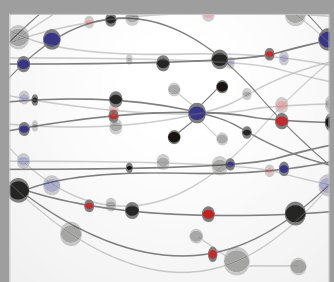

The Scientific World Journal
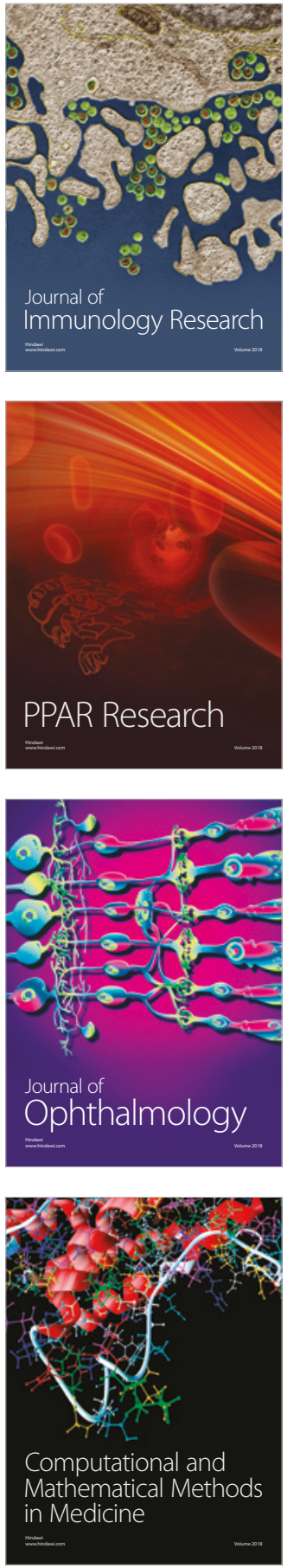

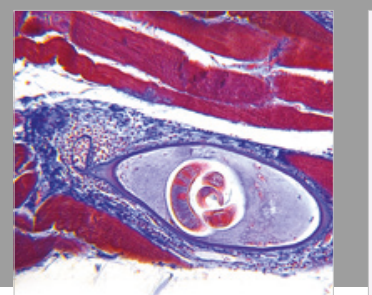

Gastroenterology Research and Practice

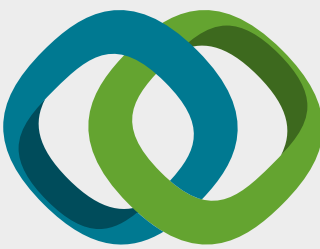

\section{Hindawi}

Submit your manuscripts at

www.hindawi.com
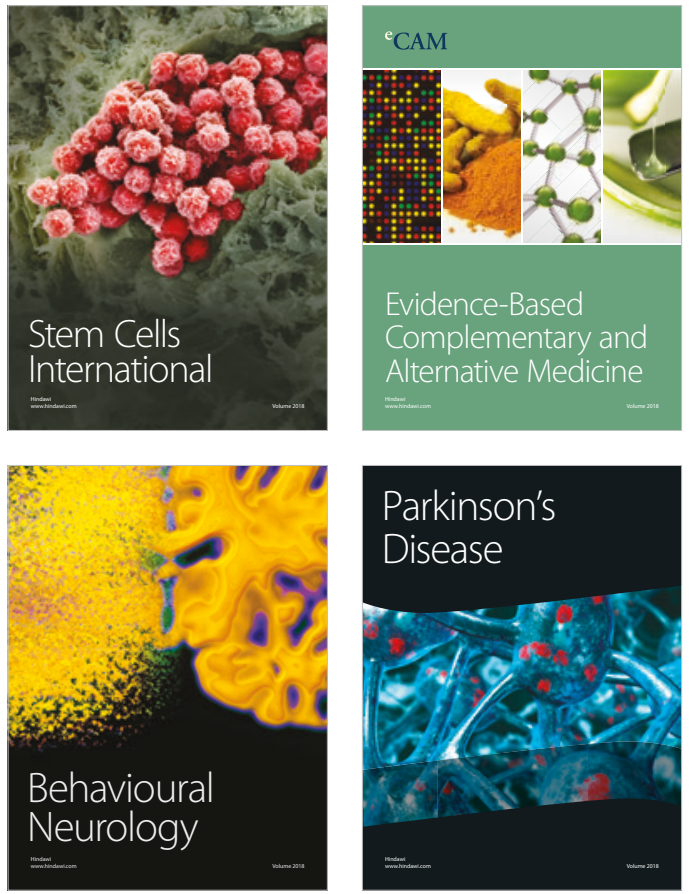

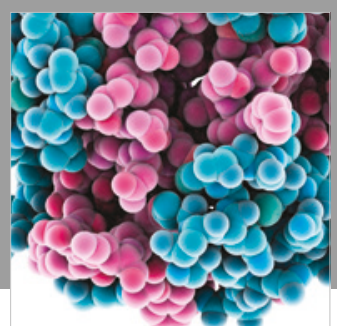

ournal of

Diabetes Research

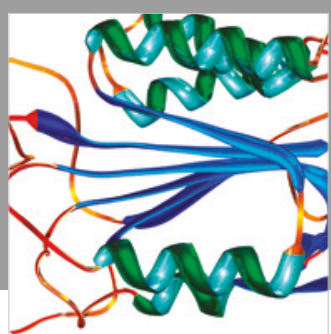

Disease Markers
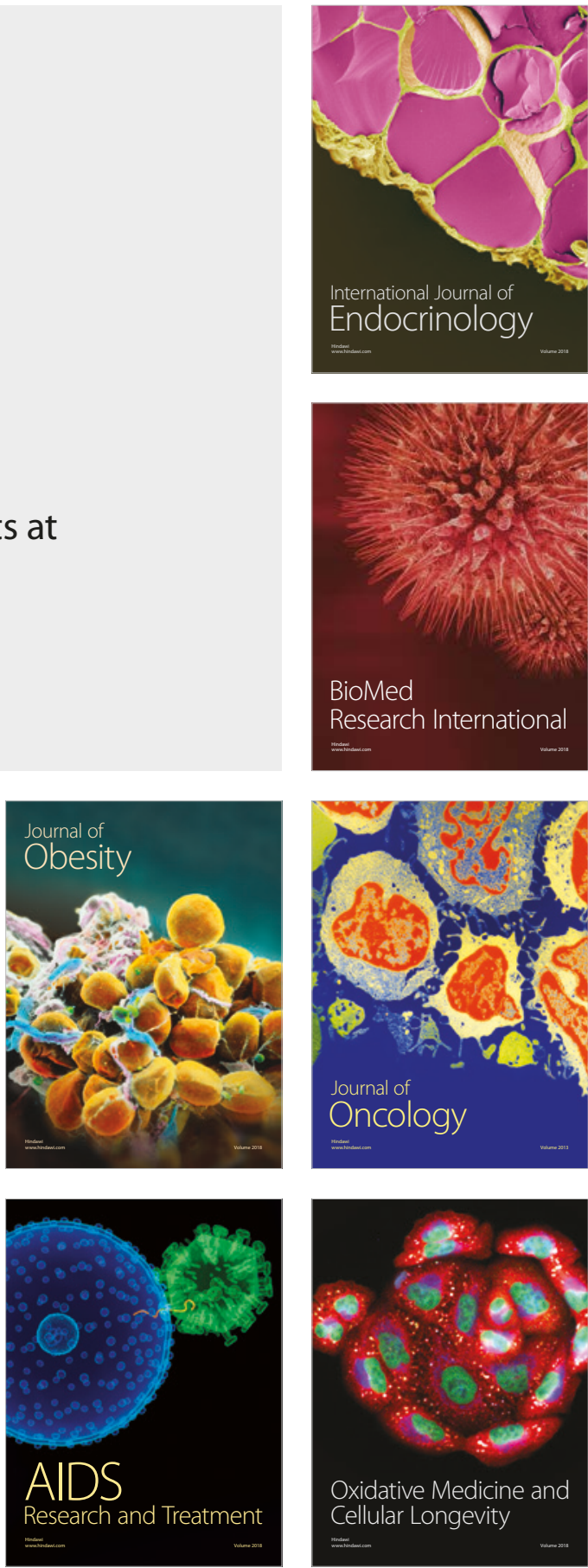